\title{
Aproximación de la interacción en salud mental desde la fisioterapia en los entornos laborales
}

\author{
Lena Yalitsa Coy Moreno ${ }^{\text {a1 }}$ \\ http://orcid.org/0000-0001-9636-1588 \\ Diana Derly Bueno Castro ${ }^{a}$ \\ https://orcid.org/0000-0002-4085-580X \\ a Universidad de la Sabana, Bogotá, Colombia.
}

Fecha de recepción: 05 de octubre de 2018

Fecha de aprobación: 04 de julio de 2019

\begin{abstract}
Resumen
La salud mental es un tema que ha tenido bastante dificultad en su reconocimiento, manejo y prevención a lo largo de los años, especialmente en el área laboral. Los trastornos mentales pueden afectar de manera significativa la salud del trabajador y por ende la productividad laboral. Esta revisión tiene como objetivo conocer sobre las herramientas evaluativas y estrategias de intervención en salud mental desde la Fisioterapia en los entornos laborales, a partir de la revisión de la literatura. Método: Estudio tipo revisión sistemática, con base en la búsqueda de artículos científicos en las bases de datos de Bireme, Medline, Pubmed, Sciencedirect y Springerlink; obteniéndose una muestra final de 20 de acuerdo con el cumplimiento de todos los criterios de inclusión, que posteriormente fueron analizados en niveles de evidencia y grado de recomendación según la metodología Oxford. Resultados: De los 20 artículos seleccionados se identificaron 2 categorías de análisis desde los objetivos de los estudios, el 1) Intervenciones para el manejo de la salud mental y 2) Herramientas evaluativas de la salud mental en los entornos laborales 3) conceptualización en el tema. Discusión: Los estudios evidencian resultados positivos de programas de salud mental realizados a través de la promoción del movimiento corporal humano en donde se evidencia diversidad, aunado a que las herramientas de evaluación son acordes al contexto de la cultura organizacional.
\end{abstract}

Palabras clave: Salud mental; fisioterapia; promoción de la salud; salud laboral

${ }^{1}$ Correo de contacto: lena.coym@gmail.com

DOI: 10.30788/RevColReh.v18.n2.2019.395

Esta obra se encuentra bajo licencia internacional CC BY 4.0 Institución editora: Escuela Colombiana de Rehabilitación 


\title{
Approximation of the interaction in mental health from the physical therapy in the work environments
}

\begin{abstract}
Mental health a subject that has not had enough recognition, management, and prevention over the years, especially in work areas. Mental disorders can significantly affect the health of the workers, and therefore reduce labor productivity in a company. This review aims to learn about the evaluation tools and intervention strategies in mental health over Physical Therapy in work environments, from the review of the literature. Method: Systematic review type study, based on the search for scientific articles in the databases of Bireme, Medline, PubMed, ScienceDirect and SpringerLink; obtaining a final sample of 20 according to the fulfillment of all the inclusion criteria, which were subsequently analyzed in levels of evidence and degree of recommendation according to the Oxford methodology. Results: Of the 20 selected articles, 2 categories of analysis were identified from the objectives of the studies, 1) Interventions for mental health management and 2) Mental health assessment tools in work environments 3) Conceptualization in the subject. Discussion: The studies show positive results of mental health programs carried out through the promotion of the human body movement where diversity is evidenced, together with the fact that the evaluation tools are in line with the organizational culture context.
\end{abstract}

Key Word: Mental health; physical therapy specialty; health promotion; occupational health

\section{Introducción.}

Según la Organización Mundial de la Salud (OMS), la salud mental se define como un "un estado de bienestar en el cual el individuo es consciente de sus propias capacidades, puede afrontar las tensiones normales de la vida, puede trabajar de forma productiva y fructífera y es capaz de hacer una contribución a su comunidad" (Bolier et al., 2014). En los últimos años, la incidencia de patologías asociadas con la salud mental se ha convertido en un problema de salud pública que va en aumento, ya que en promedio 450 millones de personas son afectadas por algún tipo de trastorno mental y casi 1 millón de personas se suicidan en el mundo cada año (Asamblea Mundial de la Salud, 2013).

Existen múltiples trastornos mentales, sin embargo se caracterizan por la combinación de alteraciones del pensamiento, de la percepción, de las emociones, de la conducta y de las relaciones con los demás. Según la OMS, la depresión y la ansiedad son los trastornos mentales más frecuentes y se encuentran entre las principales causas de discapacidad en el mundo. Frente a ello, en 2012 la Asamblea Mundial de la Salud creó un plan integral de salud mental que abarque los servicios, políticas, leyes, planes, estrategias y programas; este plan de acción es integral y multisectorial para cubrir todas las necesidades de la población (Asamblea Mundial de la Salud, 2013).

En el mundo del trabajo, dentro de los trastornos de salud mental se contemplan además de la depresión y la ansiedad, el estrés por el trabajo y casos de agotamiento identificados a través del factor de riesgo psicosocial, definido por la OIT (Organización Internacional del trabajo, 2014) como "las interacciones entre el medio ambiente del trabajo, el contenido del trabajo, las condiciones de organización y las capacidades, las necesidades y la cultura del trabajo, las consideraciones personales 
externas al trabajo que pueden tener influencia en la salud, el rendimiento del trabajo y la satisfacción laboral" (Boada-Grau \& Ficapal-Cusí, 2012).

El trabajo es un "determinante primario de la posición socioeconómica y forma un papel clave en la conexión social, el desarrollo de la identidad y la autoestima” (Reavley, Ross, Martin, LaMontagne, \& Jorm, 2014), también es entendido como un escenario importante para la promoción de la salud y la prevención de la enfermedad. El trabajo, además de abordar problemas de salud, debe permitir el abordaje de problemas relacionados con el componente individual y el extralaboral, que en ocasiones se hacen visibles en el entorno laboral (Vieco Gómez \& Abello Llanos, 2014; Wu et al., 2016; Wollesen, Menzel, Drögemüller, Hartwig, \& Mattes, 2017). La evidencia afirma que, un ambiente de trabajo psicosocial deficiente con factores de estrés laboral (a nivel individual, del grupo de trabajo o a nivel organizacional), puede aumentar el riesgo de problemas de salud mental y física, generando un gran impacto social y afectando significativamente la salud del trabajador y por ende la productividad laboral debido al ausentismo y presentismo; por ello es fundamental que las organizaciones identifiquen y modifiquen de manera temprana estos factores (Bolier et al., 2014; Reavley et al., 2014; Memish, Martin, Bartlett, Dawkins, \& Sanderson, 2017).

De acuerdo con datos de la Organización Internacional del Trabajo (OIT) los riesgos psicosociales (inseguridad laboral, el escaso control, la elevadas exigencias, el desequilibrio de recompensa por el esfuerzo realizado) y el estrés el trabajo son factores que llevan al trabajador a tener malos hábitos de vida saludable (Díaz, 2016), permitiendo un aumento en el consumo de alcohol y tabaco, alteraciones nutricionales como el sobrepeso y la obesidad, comportamientos sedentarios y alteraciones del ciclo vigilia sueño; los cuales se traducen en enfermedades no transmisibles (Nordin, Westerholm, Alfredsson, \& Åkerstedt, 2012; Kivimäki et al., 2015), trastornos musculo-esqueléticos (Deeney \& O'Sullivan, 2009; Fernández, Assunção, Silvany Neto, \& Carvalho, 2010; Oakman, Macdonald, Bartram, Keegel, \& Kinsman, 2018), síndrome de burnout, abuso de sustancias psicoactivas, depresión (Sa \& Fleming, 2008) y suicidios (Organización Mundial de la Salud, 2018).

El bienestar laboral actúa como un factor de protección para la salud mental y el funcionamiento saludable, además se ha demostrado que las intervenciones de estrés laboral que se dirigen tanto a las condiciones laborales como a las habilidades y comportamientos individuales son beneficiosas para la salud (Bolier et al., 2014; Reavley et al., 2014; Huber, Lechner, \& Wunsch, 2015). Es así como los fisioterapeutas desarrollan, a través de sus intervenciones, factores protectores de salud mental, pero para esto les es importante identificar las necesidades de salud mental. Ante este último reto es necesario conocer qué herramientas evaluativas son aplicadas desde la evidencia y cuales son de su aplicación.

Este estudio pretende indagar, a la luz de a la evidencia científica, la aproximación de la fisioterapia a aspectos que incluyen las intervenciones, las herramientas y los conceptos desde a salud mental a nivel mundial.

\section{Método.}

El presente trabajo corresponde a una revisión sistemática que se constituye en una investigación secundaria. Se partió de la evidencia disponible en los artículos publicados sobre las intervenciones, 
herramientas y conceptos desde la fisioterapia en los entornos laborales, con el fin de establecer relaciones y conclusiones generales, que muestren elementos comunes y diferenciales de los resultados señalados por los investigadores, para el periodo comprendido entre los años 2008 y 2018. La selección de los artículos se realizó de acuerdo con el flujograma que se presenta en la figura 1.

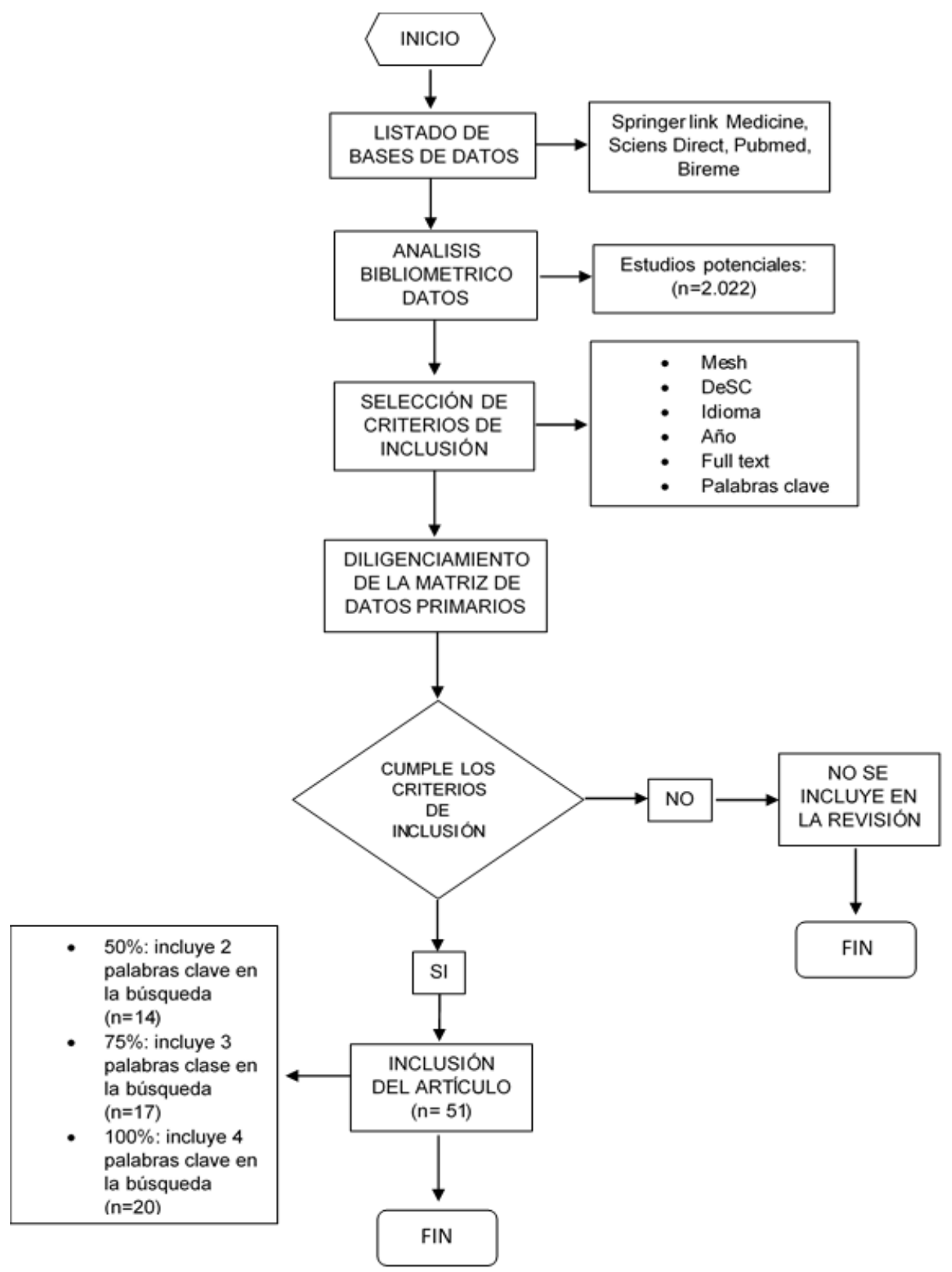

Figura 1. Flujograma de búsqueda y selección de artículos incorporados en la revisión

\section{Estrategia de búsqueda}


La búsqueda de la información fue realizada entre los meses de agosto y noviembre de 2018. Las bases de datos consultadas fueron: Pubmed, Medline, Sciencedirect, Bireme y Springerlink. Los descriptores empleados fueron "health promotion" AND "mental health" AND "workplace" AND "physical therapy" AND "occupational health"; previamente confirmadas a través de los términos DeCS y MeSH y en los idiomas inglés, español y portugués. Como criterios de inclusión se consideraron investigaciones primarias (desde ensayos clínicos hasta descriptivos) y secundarias (revisión sistemática), que determinaran cuáles son las mejores herramientas y estrategias de promoción de la salud mental que puede utilizar un fisioterapeuta en seguridad y salud en el trabajo. En cuanto a los criterios de exclusión se mencionan estudios realizados con población no trabajadores, estrategias o programas que no incluyeran el movimiento corporal humano como su eje central, experiencias individuales y artículos sin acceso completo.

\section{Extracción de datos}

Se elaboró una matriz documental que contenía los apartados de título original, autor(es), revista y año de publicación, objetivo, metodología y conclusión. Así mismo se elaboraron dos tablas que sintetizan de manera descriptiva los hallazgos de los artículos, identificándose los aportes para este estudio.

\section{Resultados}

Los resultados que se presentan a continuación se hacen con base en los estudios que cumplieron el $100 \%$ de las palabras clave de búsqueda.

\section{Datos de publicación}

Se observa la distribución de publicaciones por país y año como se muestra en la tabla 1, encontrándose que la mayoría de las publicaciones fueron realizadas en el continente europeo con 10 artículos, siendo España el país que más ha escrito sobre el tema. Para el continente asiático, Japón y Corea del sur reportan 2 estudios; en el continente americano Estados unidos fue el país con mayor publicación con 4 artículos; y en Oceanía, Australia reportó 2 publicaciones.

Tabla 1.

Publicaciones por país y año

AÑOS DE PUBLICACIÓN

\begin{tabular}{llcccc}
\hline CONTINENTE & PAÍ́S & $\mathbf{2 0 0 8 - 2 0 1 0}$ & $\mathbf{2 0 1 1 - 2 0 1 4}$ & $\mathbf{2 0 1 5 - 2 0 1 8}$ & TOTAL \\
\hline \multirow{4}{*}{ Europa } & Alemania & 1 & 0 & 1 & $\mathbf{2}$ \\
\cline { 2 - 6 } & Austria & 0 & 2 & 0 & $\mathbf{2}$ \\
\cline { 2 - 6 } & España & 0 & 1 & 2 & $\mathbf{3}$ \\
\cline { 2 - 6 } & Reino unido & 0 & 1 & 1 & $\mathbf{2}$ \\
\cline { 2 - 6 } & Suecia & 0 & 0 & 1 & $\mathbf{1}$ \\
\hline \multirow{2}{*}{ Asia } & Japón & 0 & 1 & 0 & $\mathbf{1}$ \\
\cline { 2 - 6 } & Corea del sur & 0 & & & 1 \\
\hline
\end{tabular}




\begin{tabular}{lllllc}
\hline \multirow{3}{*}{ América } & Brasil & 0 & 1 & 0 & $\mathbf{1}$ \\
\cline { 2 - 6 } & Canadá & 0 & 0 & 1 & $\mathbf{1}$ \\
\cline { 2 - 5 } & Estados Unidos & 0 & 0 & 4 & $\mathbf{4}$ \\
\hline Oceanía & Australia & 0 & 0 & 2 & $\mathbf{2}$ \\
\hline TOTAL & & $\mathbf{1}$ & $\mathbf{6}$ & $\mathbf{1 3}$ & $\mathbf{2 0}$ \\
\hline
\end{tabular}

Respecto a los años de publicación, el periodo de publicación 2015 al 2018 se encontró 13 artículos; seguidos de 6 artículos en el periodo 2011 al 2014 y 1 en el periodo comprendido entre 2008 y 2010. El 95\% de los artículos están escritos en inglés, el 5\% restante idioma en español.

\section{Distribución por nivel de evidencia y grado de recomendación}

De acuerdo con el Centre for Evidence-Based Medicine (CEBM) de Oxford (Primo, 2003), se encontró que un gran porcentaje de los estudios fueron calificados en un nivel de evidencia $1 \mathrm{~b}$ en un $25 \%$ y 2 a en un $15 \%$, lo que significa que la mayoría de los estudios son ensayos clínicos aleatorizados con intervalo de confianza estrecho, revisiones sistemáticas de estudios de cohortes, con homogeneidad y estudios de cohortes o ensayos clínicos aleatorizados de baja calidad según se observa en la tabla 2.

Tabla 2. Distribución por nivel de evidencia y grado de recomendación.

\section{Centre for Evidence-Based Medicine (CEBM) de Oxford}

\begin{tabular}{|c|c|c|c|c|c|}
\hline \multirow[b]{2}{*}{$\begin{array}{l}\text { Niveles de } \\
\text { evidencia }\end{array}$} & \multicolumn{2}{|c|}{ Artículos } & \multirow[b]{2}{*}{$\begin{array}{c}\text { Grados de } \\
\text { recomendación }\end{array}$} & \multicolumn{2}{|c|}{ Artículos } \\
\hline & Frecuencia & $\begin{array}{c}\text { Frecuencia } \\
\text { relativa } \\
\text { porcentual }\end{array}$ & & Frecuencia & $\begin{array}{c}\text { Frecuencia } \\
\text { relativa } \\
\text { porcentual }\end{array}$ \\
\hline $1 \mathrm{a}$ & 1 & $5 \%$ & $\mathbf{A}$ & 3 & $15 \%$ \\
\hline $1 \mathrm{~b}$ & 5 & $25 \%$ & & & \\
\hline $1 \mathrm{c}$ & 0 & $0 \%$ & & & \\
\hline $2 \mathbf{a}$ & 3 & $15 \%$ & B & 10 & $50 \%$ \\
\hline $2 b$ & 2 & $10 \%$ & & & \\
\hline $2 c$ & 2 & $10 \%$ & & & \\
\hline $3 \mathbf{a}$ & 2 & $10 \%$ & $\mathbf{C}$ & 5 & $25 \%$ \\
\hline $3 b$ & 2 & $10 \%$ & & & \\
\hline 4 & 1 & $5 \%$ & D & 2 & $10 \%$ \\
\hline 5 & 2 & $10 \%$ & & & \\
\hline TOTAL & 20 & $100 \%$ & TOTAL & 20 & $100 \%$ \\
\hline
\end{tabular}

Frente los grados de recomendación, se evidenció que el 50\% de los estudios se calificó en grado B, es decir que tienen una recomendación favorable. 


\section{Análisis por objetivos de estudio.}

Identificando categorías de análisis, los objetivos se describirán de acuerdo con: 1) las intervenciones en salud mental; 2) herramientas evaluativas; 3) conceptualización en salud mental, los cuales serán descritos a continuación:

Intervenciones en salud mental. La tabla 3 relaciona los estudios que demarcaron intervenciones

Tabla 3.

Intervenciones en salud mental

\begin{tabular}{ll}
\hline \multicolumn{1}{c}{ Objetivos de las intervenciones en salud mental } & \multicolumn{2}{c}{ Autores } \\
\hline $\begin{array}{l}\text { Creación de programas en torno al bienestar y la promoción de } \\
\text { la salud en el lugar de trabajo con el objetivo de reducir el }\end{array}$ & $\begin{array}{l}\text { (Ammendolia et al., 2016; Wong } \\
\text { ausentismo. }\end{array}$ \\
$\begin{array}{ll}\text { Intervenciones en las cuales se distinguen, el uso de } \\
\text { estiramientos para el manejo de la ansiedad y el estrés. }\end{array}$ & (Montero-Marín, Asún, Estrada- \\
\end{tabular}

Ejercicio ecológico sobre los indicadores psicológicos y (Calogiuri et al., 2016)

fisiológicos del estrés.

Descanso activo en las relaciones personales, actividad física (Michishita et al., 2016)

y gestión de la capacidad de trabajo.

Programa de mejora de la salud y bienestar en el lugar de trabajo a través de ejercicio y nutrición.

(Coffeng et al., 2014; Emerson, Merrill, Shedd, Bilder, \& Siddarth, 2017)

Programa de incentivos financieros individuales y en equipo

(Losina et al., 2017; para aumentar la actividad física entre empleados sedentarios. LaMontagne et al., 2014)

Desde programas de Ergonomía para prevención del estrés, (Grensman et al., 2018; bajo la utilización en yoga para mejorar el autocuidado y Alexander, Rollins, Walker, reducir el agotamiento.

Wong, \& Pennings, 2015)

El desafío de 10.000 pasos para el manejo de la salud mental y bienestar.

(Hallam, Bilsborough, \& de Courten, 2018)

(Grensman et al., 2018)

Uso de terapia cognitiva basada en la atención plena (TCAM) o la terapia cognitivo conductual (TCC) en personas con agotamiento.

(Kim et al., 2014)

Uso de una estrategia biopsicosocial de manera individual.

Ejercicio ecológico sobre los indicadores psicológicos y (Calogiuri et al., 2016) fisiológicos del estrés 
Herramientas evaluativas. Se encontró que del 100\% de los estudios analizados, el $60 \%$ aplicó un tipo de escala de evaluación relacionada con salud mental, como se observa en la tabla 4.

Tabla 4.

Herramientas evaluativas en salud mental

\begin{tabular}{|c|c|}
\hline Características evaluadas & Autores \\
\hline $\begin{array}{ll}\text { - } & \text { Dolor musculo esquelético, } \\
\text { - } & \text { Bienestar físico. } \\
\text { - } & \text { Salud mental. } \\
\text { - } & \text { Actividad física. } \\
\text { - } & \text { Estrés }\end{array}$ & (O’Connor, Neff, \& Pitman, 2018) \\
\hline Calidad de vida personal y laboral & $\begin{array}{l}\text { (Kobayashi, Kaneyoshi, Yokota, \& Kawakami, } \\
\text { 2008), }\end{array}$ \\
\hline $\begin{array}{l}\text { Evaluación del sueño, del agotamiento, } \\
\text { evaluación del síndrome de Burnout, }\end{array}$ & $\begin{array}{l}\text { (Greco, Magnago, Urbanetto, Luz, \& } \\
\text { Prochnow, 2015) }\end{array}$ \\
\hline Habilidades laborales y violencia laboral & (Lancman, Ghirardi, Castro, \& Tuacek, 2009) \\
\hline $\begin{array}{l}\text { Escalas de evaluación para evaluar el impacto } \\
\text { de sus intervenciones. }\end{array}$ & $\begin{array}{l}\text { (Urbanetto et al., 2011; (Oakman et al., 2018; } \\
\text { Jarman, Martin, Venn, Otahal, \& Sanderson, } \\
\text { 2015) }\end{array}$ \\
\hline
\end{tabular}

Estudios teóricos. Del total de la búsqueda se encontraron cuatro estudios teóricos, los cuales se detallan en la tabla 5 .

Tabla 5.

Estudios teóricos en salud mental

\begin{tabular}{ll}
\hline \multicolumn{1}{c}{ Tema } & \multicolumn{1}{c}{ Autores } \\
\hline Intervenciones a nivel primario, secundario y terciario. & (Joyce et al., 2016) \\
Estrategias para crear entornos laborales saludables. & $\begin{array}{l}\text { (Pinkstaff, McNeil, Arena, } \\
\text { \& Cahalin, 2017) }\end{array}$ \\
Pautas de detección de problemas de salud mental para empleadores & (Brand et al., 2017) \\
Gestión de los problemas de salud mental en el lugar de trabajo. & $\begin{array}{l}\text { (Goldgruber \& Ahrens, } \\
\text { 2010) }\end{array}$ \\
\hline
\end{tabular}


Distribución según la metodología de los estudios. Respecto a la metodología se tuvo en cuenta el tipo de estudio, número de participantes por estudio y los sectores económicos en los que se aplicó.

De acuerdo con el diseño y tipo de estudio, el 25\% que corresponden a estudios experimentales (preexperimentales, de preprueba y posprueba con grupo control, cuasiexperimentales); con el mismo porcentaje, $20 \%$ se encuentran los estudios de tipo descriptivos (transversales, correlacionales y longitudinales), ensayos clínicos aleatorizados ciego; el 15\% son de tipo revisiones narrativas de la literatura; finalmente con el mismo porcentaje equivalente al $10 \%$ se encuentran estudios de tipo revisiones sistemáticas y estudios cualitativos.

Sobre la distribución por muestra, se establecieron rangos por número de personas participantes del estudio de la siguiente manera: el 75\% se ubicó en el rango de 0 a 500, seguido de un $10 \%$ en el rango de 501 a 1000 y de 1000 a 10000 y por último, un 5\% equivalente a 10000 personas o más.

Teniendo en cuenta el sector económico en el que se realizaron las investigaciones, se evidencia que los sectores en los que se encontró mayor relación con la salud mental fue el sector salud (enfermeros, auxiliares de enfermería, médicos, residentes, fisioterapeutas, odontólogos), el sector educativo, agricultura (minería, forestación), industria (manufactura, consultoría), transporte, financiero y de riesgo público (bomberos, policías y transporte).

Respecto al análisis según los resultados de los estudios, se tuvo en cuenta si los artículos arrojaron resultados con significancia estadística, si se obtenían resultados favorables o desfavorables al realizar el estudio y si estos llegaban a ser no conclusivos; otros aspectos fueron si los resultados resultaron desfavorables y otros si no tuvieron significancia estadística. Frente a estas categorías se encontró que el $31 \%$ obtuvieron resultados favorables, el $27 \%$ de los estudios obtuvieron resultados con significancia estadística, $23 \%$ fueron estudios no conclusivos, $12 \%$ resultados desfavorables y $8 \%$ sin significancia estadística.

\section{Discusión}

Desde la academia, siempre se enseña al estudiante que debe hacer un abordaje fisioterapéutico del paciente o usuario de forma holística, en el que se incluya un análisis desde todas las dimensiones del ser, sin embargo, es frecuente que se priorize el movimiento corporal humano desde el componente biológico, perdiéndose de vista, el análisis y manejo de otros factores que pueden afectarlo.

De acuerdo con lo documentado "los trastornos mentales pueden afectar directamente el estado físico o viceversa"(Catalán Matamoros, 2009). Esto dio paso a que en Europa, profesionales en fisioterapia tuvieran la visión y la misión de documentar e incursionar en aquellas intervenciones terapéuticas que se podían aplicar para el manejo de patologías asociadas al componente neuropsicológico. En ese recorrido se ha permitido justificar la participación de los fisioterapeutas, como parte activa y fundamental dentro de un equipo multidisciplinar para el manejo de la salud mental, con el objetivo de mejorar los síntomas y la calidad de vida de la persona (Probst, 2017) y por ende sus efectos sobre la corporeidad y la corporalidad. 
En Colombia, el rol del fisioterapeuta en salud mental aun es muy reciente, sin embargo, ha logrado un importante reconocimiento y han permitido cambios de paradigmas frente al desempeño profesional. En el marco de esta nueva área de estudio y desempeño, esta investigación buscó indagar como el manejo de salud mental puede ser una de las acciones a realizar como fisioterapeutas en el mundo del trabajo, siendo esta comprendida como una parte importe y significativa del ciclo vital de la persona, la cual puede generar un impacto positivo o negativo sobre su estado de salud y por ende influir significativamente en sus demás dimensiones.

Es así como las estadísticas indican que la depresión y el estrés se han convertido en la primera y segunda causa de mayor pérdida de productividad laboral por ausentismo, asociadas al diagnóstico de patologías de salud mental, musculoesqueléticas, cardiovasculares y diabetes, cáncer y gripe (Ammendolia et al., 2016).

En este sentido, las organizaciones que en la actualidad han despertado un sentido de responsabilidad social frente a todos sus colaboradores, en donde el trabajador es identificado como uno de los más importante; han procurado generar e implementar programas y estrategias que buscan mitigar o proteger a esta población del impacto que pueda tener sobre el estado de salud, las condiciones psicosociales a las cuales se ve expuesto en el desarrollo de la labor.

La evidencia encontrada documenta diversas intervenciones, como son la realización de la actividad física y otras técnicas que propician el movimiento, para disminuir significativamente los niveles de estrés, depresión, ansiedad y así mismo fomentar el bienestar en el lugar de trabajo (Hallam et al., 2018; Kim et al., 2014; Montero-Marín et al., 2013), el bienestar emocional (Alexander et al., 2015) y el bienestar físico, respecto a la función cognitiva, sueño y autocuidado (Alexander et al., 2015; Grensman et al., 2018).

También se han documentado intervenciones cognitivo-conductuales y técnicas de relajación como las más utilizadas para el manejo del estrés, que se potencializan al combinarlas con el rediseño del trabajo, la reducción de las demandas de trabajo, una mejor comunicación y el desarrollo de habilidades para el manejo de conflictos (Goldgruber \& Ahrens, 2010; Jay et al., 2015).

Los programas de entrenamiento participativo orientado a la acción (PAOT), cuyo objetivo es el entrenamiento integral de manejo del estrés laboral, fue también efectivo para reducirlo, mejorando en los trabajadores administrativos, sus habilidades laborales, el apoyo mutuo y el estrés psicológico; y en los trabajadores operativos, mejora también la salud mental y las habilidades laborales (Kim et al., 2014).

Del análisis de las investigaciones consultadas, se identificó que las profesiones de Enfermería y educación (docentes), presentan mayor afectación de la salud mental por la carga psicosocial que enfrentan asociada al tipo de población que manejan. En estos grupos, se documentaron intervenciones que permitió mejorar la calidad de vida y mayor satisfacción laboral, debido a la disminución de los índices de estrés y de esfuerzo físico (Márquez Membrive et al., 2011; (Pinkstaff et al., 2017); del mismo modo, la creación de un programa de resiliencia para reducir el estrés fisiológico y psicológico mostró mejora en mecanismos de sobrellevarlo, mejorando también el manejo de relaciones familiares, desempeño laboral (Prochnow et al., 2013) y habilidades interpersonales (McCraty \& Atkinson, 2012). 
Sin embargo, la puesta en marcha de acciones de promoción y prevención en salud mental en los entornos laborales, se considera como una tarea exclusiva de los profesionales en psicología y el abordaje en ocasiones es difícil, por múltiples razones. Se resalta la estigmatización que se genera, la falta de conciencia por parte de la organización sobre el impacto sobre una buena salud mental en el desarrollo de la labor y en la misma medida, la falta de conciencia en el autocuidado por parte del trabajador.

Se sugiere que se continúen documentando las herramientas evaluativas, los programa y estrategias que desde fisioterapia se pueden generar e implementar para contribuir no solo con una salud física, sino que justamente a través de ella se pueda influir significativamente en la salud mental en las diferentes etapas del ciclo vital, ampliando nuestras competencias profesionales específicas y así mismo, ampliar los criterios de desempeño en salud y trabajo.

\section{Conclusiones.}

Los estudios han demostrado consistentemente los resultados positivos de programas de salud mental, sin embargo, las metas de los programas deben ir acorde con la estructura organizacional y la cultura del lugar de trabajo bajo el uso de herramientas de evaluación apropiadas.

Las revistas europeas lideran las publicaciones en salud mental a nivel mundial. Los años de mayor publicación obedecen de 2015 a 2018. El nivel de evidencia que predomino en los estudios es 1 b el grado de recomendación, grado $\mathrm{B}$ y en el idioma inglés.

Se concluye además por los objetivos de los estudios que frente a las intervenciones la mayoría favorecen el ejercicio y de forma novedosa su denominación como ejercicio ecológico que va desde el yoga, programas de pasos e incentivos económicos por su realización. Por otra parte, frente a las herramientas, las caracterizaciones en salud mental tuvieron un papel preponderante en análisis y evaluación de la calidad de vida y de síndromes relacionados con problemas de salud mental como el Burnout. Frente a los conceptos se identifican los artículos de detección de problemas de salud mental, estrategias de gestión de estas y promoción de entornos laborales saludables.

Es necesario seguir ahondando en estudios que nos de la mayor aproximación al enfoque de la fisioterapia en salud mental.

\section{Agradecimientos}

Las autoras agradecen el valioso apoyo de las estudiantes del programa de Fisioterapia de la Universidad de La Sabana de noveno semestre - práctica de profundización I en Seguridad y Salud en el trabajo durante el periodo académico 2018-2,

\section{Conflicto de Intereses}

Las autoras declaran no presentar ninguna relación de interés comercial o personal dentro del marco de la realización del manuscrito y la investigación que condujo a la producción del manuscrito.

\section{Colaboraciones}

Todas las autoras han contribuido intelectualmente en la elaboración del documento. 


\section{Referencias}

Alexander, G. K., Rollins, K., Walker, D., Wong, L., \& Pennings, J. (2015). Yoga for self-care and burnout prevention among nurses. Workplace health \& safety, 63(10), 462-470. Doi: $10.1177 / 2165079915596102$

Ammendolia, C., Côté, P., Cancelliere, C., Cassidy, J. D., Hartvigsen, J., Boyle, E., ... Amick, B. (2016). Healthy and productive workers: using intervention mapping to design a workplace health promotion and wellness program to improve presenteeism. BMC Public Health, 16(1), 1190. Doi: 10.1186/s12889-016-3843-x

Asamblea Mundial e la Salud. (2013). Plan de acción integral sobre salud mental 2013-2020.

Boada-Grau, J., \& Ficapal-Cusí, P. (2012). Salud y trabajo. Los nuevos y emergentes riesgos psicosociales (Vol. 206). Editorial UOC.

Bolier, L., Ketelaar, S. M., Nieuwenhuijsen, K., Smeets, O., Gärtner, F. R., \& Sluiter, J. K. (2014). Workplace mental health promotion online to enhance well-being of nurses and allied health professionals: A cluster-randomized controlled trial. Internet interventions, 1(4), 196-204. Doi: 10.1016/j.invent.2014.10.002

Brand, S. L., Coon, J. T., Fleming, L. E., Carroll, L., Bethel, A., \& Wyatt, K. (2017). Whole-system approaches to improving the health and wellbeing of healthcare workers: A systematic review. PloS one, 12(12), e0188418. Doi: 10.1371/journal.pone.0188418

Calogiuri, G., Evensen, K., Weydahl, A., Andersson, K., Patil, G., Ihlebæk, C., \& Raanaas, R. K. (2016). Green exercise as a workplace intervention to reduce job stress. Results from a pilot study. Work, 53(1), 99-111. Doi: 10.3233/wor-152219

Catalán Matamoros, D. (2009). La Fisioterapia en Salud Mental: su efectividad y situación actual. Fisioterapia, 31(5), 175-176. Doi: 10.1016/j.ft.2009.09.001

Coffeng, J. K., Boot, C. R., Duijts, S. F., Twisk, J. W., van Mechelen, W., \& Hendriksen, I. J. (2014). Effectiveness of a worksite social \& physical environment intervention on need for recovery, physical activity and relaxation; results of a randomized controlled trial. PloS one, 9(12), e114860. Doi: 10.1371/journal.pone.0114860

Deeney, C., \& O’Sullivan, L. (2009). Work related psychosocial risks and musculoskeletal disorders: potential risk factors, causation and evaluation methods. Work, 34(2), 239-248.

Díaz, D. Z. (2016). Estrés en el trabajo: un reto colectivo. Artículos de Argentina, Colombia y Chile Reseñas de Libros Facultad Tecnólogica, 60.

Emerson, N., Merrill, D., Shedd, K., Bilder, R., \& Siddarth, P. (2017). Effects of an employee exercise programme on mental health. Occupational Medicine, 67(2), 128-134. Doi: 10.1093/occmed/kqw120

Fernandes, R. de C. P., Assunção, A. Á., Silvany Neto, A. M., \& Carvalho, F. M. (2010). Musculoskeletal disorders among workers in plastic manufacturing plants. Revista brasileira de epidemiologia, 13(1), 11-20. Doi: 10.1590/s1415-790x2010000100002

Goldgruber, J., \& Ahrens, D. (2010). Effectiveness of workplace health promotion and primary prevention interventions: a review. Journal of Public Health, 18(1), 75-88. Doi: 10.1007/s10389-009-0282-5 
Greco, P. B. T., Magnago, T. S. B. de S., Urbanetto, J. de S., Luz, E. M. F. da, \& Prochnow, A. (2015). Prevalence of minor psychiatric disorders in socio-educational agents in the state of Rio Grande do Sul. Revista brasileira de enfermagem, 68(1), 93-101. Doi: 10.1590/0034-7167.2015680113p

Grensman, A., Acharya, B. D., Wändell, P., Nilsson, G. H., Falkenberg, T., Sundin, Ö., \& Werner, S. (2018). Effect of traditional yoga, mindfulness-based cognitive therapy, and cognitive behavioral therapy, on health related quality of life: a randomized controlled trial on patients on sick leave because of burnout. BMC complementary and alternative medicine, 18(1), 80. Doi: 10.1186/s12906-018-2141-9

Hallam, K., Bilsborough, S., \& de Courten, M. (2018). "Happy feet": evaluating the benefits of a 100-day 10,000 step challenge on mental health and wellbeing. BMC psychiatry, 18(1), 19. Doi: 10.1186/s12888-018-1609-y

Huber, M., Lechner, M., \& Wunsch, C. (2015). Workplace health promotion and labour market performance of employees. Journal of health economics, 43, 170-189. Doi: 10.1016/j.jhealeco.2015.07.002

Jarman, L., Martin, A., Venn, A., Otahal, P., \& Sanderson, K. (2015). Does workplace health promotion contribute to job stress reduction? Three-year findings from Partnering Healthy@ Work. BMC public health, 15(1), 1293. Doi: 10.1186/s12889-015-2625-1

Jay, K., Brandt, M., Hansen, K., Sundstrup, E., Jakobsen, M. D., Schraefel, M., ... Andersen, L. L. (2015). Effect of individually tailored biopsychosocial workplace interventions on chronic musculoskeletal pain and stress among laboratory technicians: randomized controlled trial. Pain Physician, 18(5), 459-471. Doi: 10.1186/1471-2474-15-444

Joyce, S., Modini, M., Christensen, H., Mykletun, A., Bryant, R., Mitchell, P. B., \& Harvey, S. B. (2016). Workplace interventions for common mental disorders: a systematic meta-review. Psychological Medicine, 46(4), 683-697. Doi: 10.1017/s0033291715002408

Kim, S.-A., Suh, C., Park, M.-H., Kim, K., Lee, C.-K., Son, B.-C., ... Kang, K. (2014). Effectiveness of a comprehensive stress management program to reduce work-related stress in a medium-sized enterprise. Annals of occupational and environmental medicine, 26(1), 4. Doi: 10.1186/2052-437426-4

Kivimäki, M., Virtanen, M., Kawachi, I., Nyberg, S. T., Alfredsson, L., Batty, G. D., ... Burr, H. (2015). Long working hours, socioeconomic status, and the risk of incident type 2 diabetes: a meta-analysis of published and unpublished data from 222120 individuals. The lancet Diabetes \& endocrinology, 3(1), 27-34. Doi: 10.1016/s2213-8587(14)70178-0

Kobayashi, Y., Kaneyoshi, A., Yokota, A., \& Kawakami, N. (2008). Effects of a worker participatory program for improving work environments on job stressors and mental health among workers: a controlled trial. Journal of occupational health, 50(6), 455-470. Doi: 10.1539/joh.17166

LaMontagne, A. D., Martin, A., Page, K. M., Reavley, N. J., Noblet, A. J., Milner, A. J., ... Smith, P. M. (2014). Workplace mental health: developing an integrated intervention approach. BMC psychiatry, 14(1), 131. Doi: 10.1186/1471-244x-14-131

Lancman, S., Ghirardi, M. I. G., Castro, E. D. de, \& Tuacek, T. A. (2009). Repercussions of violence on the mental health of workers of the Family Health Program. Revista de saude publica, 43(4), 682688. Doi: 10.1590/s0034-89102009005000036

Losina, E., Smith, S. R., Usiskin, I. M., Klara, K. M., Michl, G. L., Deshpande, B. R., ... Katz, J. N. (2017). Implementation of a workplace intervention using financial rewards to promote adherence 
to physical activity guidelines: a feasibility study. BMC public health, 17(1), 921. Doi: 10.1186/s12889-017-4931-2

Márquez Membrive, J., Granero-Molina, J., Solvas Salmerón, M. J., Fernández-Sola, C., Rodríguez López, C. M., \& Parrón Carreño, T. (2011). Quality of life in perimenopausal women working in the health and educational system. Revista latino-americana de enfermagem, 19(6), 1314-1321. Doi: 10.1590/s0104-11692011000600006

\section{Notas:}

El presente artículo se derivó de la investigación titulada "Programas de promoción de la salud mental desde el movimiento corporal humano en los entornos laborales de las empresas" convenio de práctica en Salud y Seguridad en el Trabajo SST, Universidad de La Sabana.

\section{Información de autores:}

\section{Lena Yalitsa Coy Moreno}

Fisioterapeuta, MSc. en Magister en Morfología Humana

Universidad de la Sabana, Bogotá, Colombia

Universidad Agustiniana

Escuela Colombiana de Carreras Industriales

lena.coym@gmail.com

http://orcid.org/0000-0001-9636-1588

\section{Diana Derly Bueno Castro}

Fisioterapeuta, MSc. en Seguridad y Salud en el trabajo

Universidad de la Sabana, Bogotá, Colombia

diana.bueno@unisabana.edu.co

https://orcid.org/0000-0002-4085-580X 